\title{
Tecnura
}

\section{La ética en la formación del ingeniero de minas: representaciones sociales de actores educativos}

\author{
Ethics in the formation of the mining engineer: \\ social representations of educational actors
}

\author{
Raimundo Alonso Pérez Gómez*, Audin Aloiso Gamboa Suárez**, \\ César Augusto Hernández Suárez***
}

Fecha de recepción: 1 de marzo de 2014

Fecha de aceptación: 19 de enero de 2015

Financiamiento: Fondo de Investigaciones de la Universidad Francisco de Paula Santander (Cúcuta - Colombia)

Citation / Para citar este artículo: Pérez Gómez, R. A., Gamboa Suárez, A. A., \& Hernández Suárez, C. A. (2015). La ética en la formación del ingeniero de minas: representaciones sociales de actores educativos. Revista Tecnura, 19(44), 201-208. doi:http://dx.doi.org/10.14483/udistrital.jour.tecnura.2015.2.a15

\section{Resumen}

El presente artículo muestra los resultados de una investigación cualitativa que buscó comprender las representaciones sociales de docentes y estudiantes sobre la ética en una institución de educación superior en Cúcuta, Colombia. Se utilizó el método hermenéutico, lo cual posibilitó comprender un tipo de conocimiento que se construye en el diálogo, la interacción, la intersubjetividad y la cotidianidad. La muestra fue intencional y la conformaron 25 estudiantes y 2 profesores. Los resultados del estudio evidencian que para los actores educativos la ética está relacionada con los valores de los sujetos, los cuales son aprendidos del entorno. Relacionan la ética con la sociedad y la definen como una ciencia que les da a las personas ciertos parámetros que deben seguir para cumplir con las buenas acciones dentro de la sociedad.
Palabras clave: ética profesional, formación humanística, práctica moral, representaciones sociales.

\begin{abstract}
This article shows the results of a qualitative research aimed to understand the social representations of teachers and students concerning ethics in an institution of higher education in Cúcuta, Colombia. The hermeneutic method was used which allowed to understanding a kind of knowledge that is built on dialogue, interaction, intersubjectivity and everyday life. The sample was intentional and formed by 25 students and 2 teachers. The study results show that for the educational actors ethics is related to the values of the subjects, which are learned from the environment. They relate ethics to
\end{abstract}

* Ingeniero en Minas, especialista en Gerencia de Empresas, especialista en Derecho Ambiental, magíster en Práctica Pedagógica. Docente de la Universidad Francisco de Paula Santander. Cúcuta, Colombia. Contacto: planingminas@ufps.edu.co

** Licenciado en Música, magíster en Pedagogía, candidato a doctor en Ciencias de la Educación; docente de la Universidad Francisco de Paula Santander. Cúcuta, Colombia. Contacto: audingamboa@ufps.edu.co

*** Licenciado en Matemáticas y Computación, especialista en Computación para la Docencia, especialista en Práctica Pedagógica Universitaria, magíster en Enseñanza de las Ciencias Básicas - Matemáticas, doctorando en Educación. Docente de la Universidad Francisco de Paula Santander. Cúcuta, Colombia. Contacto: cesaraugusto@ufps.edu.co 
society and define it as a science that gives people certain parameters that must be followed to comply with good actions within society.
Keywords: humanistic formation, moral practice, professional ethics, social representations.

\section{INTRODUCCIÓN}

La sociedad actual ha sido calificada como una sociedad de consumo, cientificista, tecnócrata, sociedad de masas en la que por diversos factores el hombre se ha convertido en un ser estandarizado y despersonalizado. En esta sociedad el desarrollo no se contempla en forma integral y holístico sino parcial, en el que priman la tecnología, lo científico, lo económico, lo urbano y lo individual. Pocos conciben y trabajan en la tónica de un auténtico desarrollo integral en el que se conceda la prioridad a lo humano-social, que se subordinen la ciencia, la tecnología, la economía, la administración y, en general, todas las disciplinas aplicadas.

La educación superior en nuestro medio, como lo plantean Restrepo, Amésquita, Arenas y Castañeda (2006), viene desempeñando un papel secundario en el componente humanista de los programas académicos. La educación está orientada al desarrollo de las destrezas, pero se ha descuidado el aspecto más importante que es la formación humana.

En este sentido, la ética se constituye en elemento fundamental de la formación humanista del profesional y ciudadano que requiere la sociedad actual, dado que contribuye a la asimilación y apropiación de normas y patrones de conductas que regulan las relaciones entre las personas, constituyendo un sistema de valores universales e individuales que le permite insertarse activamente en su contexto, desde su propio perfil ocupacional (Pérez, 2006).

Su inclusión en el ámbito educativo, sin duda, debe realizarse porque la formación humanista constituye uno de los retos de la educación que se ofrece en las universidades, ya que es necesario que los profesionales, en tanto intermediarios entre la ciencia, la tecnología y las humanidades respecto de la sociedad, sean conscientes de las posibilidades de utilización del conocimiento teórico y práctico en función del bienestar social (De Lira, 2006). De igual forma, es una herramienta importante para que en el proceso educativo (además de las habilidades de saber y saber hacer), se pueda considerar el desarrollo de otras habilidades como la promoción de valores y la ética, tendientes a una formación integral, que permita una visión global de las condiciones del ámbito económico, político y social dentro del cual desarrolla su profesión (Agüero, 2008).

La ética tiene sentido en relación con los grupos humanos ya que es en ellos donde la persona estructura sus conceptos normativos y sus prácticas morales. La ética se construye mediante la interacción de sus individuos.

En el contexto kantiano, la ética tiene como aspiración el bien del sujeto en sí y de la comunidad en la cual el individuo interactúa con base en las acciones bajo la norma de las máximas universalizables y de la voluntad como ejercicio de la libertad (Kant, 2006). De igual manera, en los trabajos de Kohlberg, Power y Higgins (2008) se puede evidenciar que el ejercicio libre de tal voluntad se va estructurando mediante el análisis de los dilemas morales y situaciones reales a nivel escolar con el fin de alcanzar una comunidad normativa en la cual cada individuo valora las instituciones en sí mismas y estas a su vez imponen sus normas y sus responsabilidades al grupo entero.

La ética es esencialmente social y su proceso de formación surge a partir de la universalización de las costumbres que mejoran las condiciones de bienestar de los grupos. No obstante, la declaración de los códigos de comportamiento no es suficiente para lograr una comunidad con cultura 
moral; es necesario que cada individuo haya interiorizado el imperativo categórico con el cual valorará sus acciones en función de su propio bienestar y el de su grupo. No puede entonces concebirse un individuo cuyas acciones estén determinadas a lograr su bienestar usufructuando el bienestar de la sociedad, ni tampoco esta puede determinar su bienestar a costa de alguno de sus miembros.

La práctica minera es un buen ejemplo de la relación entre lo social y lo individual. Es interesante analizar que la propiedad privada cede paso a la propiedad pública en lo referente a los bienes obtenidos del subsuelo. El Código colombiano de Minas, ley 685 de 2001, en el artículo 5 expresa que los minerales de cualquier clase y ubicación yacentes en el suelo o el subsuelo son de la exclusiva propiedad del Estado, sin consideración a que la propiedad, posesión o tenencia de los correspondientes terrenos sean de otras entidades públicas, de particulares o comunidades o grupos. Al analizar el fin de la ética como una construcción de la armonía y de la convivencia social como la gran aspiración de todos los grupos humanos, es posible encontrar un sustento a la legislación mencionada y fundamentar el principio ético según el cual el bien social prima sobre el bien particular.

De este modo, este trabajo pretende comprender los discursos de los actores educativos referente a los elementos axiológicos de formación en la universidad que dan cuenta de la fundamentación en los valores necesarios para la generación de un profesional autónomo, integral, con la sensibilidad social para asumir el rol y compromiso en los procesos de concertación, convivencia, tolerancia y paz que con urgencia requiere nuestro país en particular, y en general el mundo globalizado actual.

\section{METODOLOGÍA}

El presente estudio es de enfoque cualitativo-hermenéutico, pues intenta hacer una aproximación global de las situaciones sociales para explorarlas, describirlas y comprenderlas (Bonilla \& Rodríguez, 2005).
La exploración de las representaciones de los estudiantes y profesores del plan de estudios de Ingeniería de Minas de la Universidad Francisco de Paula Santander sobre el aprendizaje y la enseñanza de la ética posibilita un tipo de conocimiento que se construye en el diálogo, la interacción, la intersubjetividad y la cotidianidad. En ese sentido, los estudios de orden cualitativo reivindican las realidades subjetiva e intersubjetiva como objetos legítimos de conocimiento científico y la vida cotidiana, como el escenario básico de construcción, constitución y desarrollo de los distintos planos que configuran e integran dichas realidades como dimensiones específicas del mundo humano. Se considera que la investigación cualitativa constituye la metodología apropiada para comprender las formas como los estudiantes y docentes participantes en este estudio, conciben el aprendizaje y la enseñanza de la ética.

La técnica de recolección de información fue la entrevista en profundidad que sigue el modelo de plática entre iguales, encuentros reiterados cara a cara entre el investigador y los informantes (Taylor \& Bodgan, 1990), lo cual permitió indagar las representaciones sociales sobre ética de los actores educativos.

La muestra fue intencional y estuvo determinada por 25 estudiantes; 5 de género masculino y 20 de género femenino que cursaban la asignatura de ética profesional durante el segundo semestre de 2012, y 2 profesores que orientan esta asignatura en la Universidad Francisco de Paula Santander (Cúcuta).

Para el análisis de la información se utilizó la teoría de Strauss y Corbin (2002) que consiste en "hacer las conceptualizaciones, definir las categorías y desarrollarlas en términos de sus propiedades y dimensiones para luego relacionarlas por medio de hipótesis o afirmaciones que establezcan relaciones" (p. 133).

Con apoyo del software ATLAS.Ti 6.0 se hizo la división de contenidos narrativos en unidades hermenéuticas, efectuando simultáneamente triangulación de fuentes. Posteriormente se procedió a la clasificación de la información existente en las 
unidades hermenéuticas con relación a las subcategorías realizando la codificación axial (Muñóz \& Gamboa, 2012).

\section{CONCLUSIONES Y DISCUSIÓN}

A continuación se mostrarán los resultados del estudio interpretados desde tres categorías de análisis: una visión de la ética en el campo profesional, factor de inspiración para hacer siempre lo correcto y conjunto de valores y principios para la convivencia en sociedad.

\section{Una visión de la ética en el campo profesional}

Las representaciones sociales sobre la ética en estudiantes dan cuenta de esta como una norma primordial desde los procesos educativos y propiamente la norma ética concreta de sus carreras. Surgen voces que manifiestan "es lo que nos va a medir el desempeño porque podemos ser muy buenos profesionales, podemos saber mucho, pero igual, si no tenemos ética, si no tenemos criterio es muy difícil salir adelante en un ámbito laboral complejo".

En este mismo sentido, un docente consideró que "la ética se manifiesta en aplicar flexibilidad, comprensión, receptividad y buen juicio en el quehacer"; así mismo, expresó que "la inflexibilidad de las normas debe ceder a la consideración de las personas sin llegar a la permisividad", y sustenta estas aseveraciones argumentando que "trato de hacer las cosas correctamente, trato en lo posible de apegarme a las normas, de repente hay algunos momentos en que la normas son inflexibles y le dan importancia al procedimiento, a la fecha, a la letra más que a la persona; trato de conciliar eso para que el ser humano siempre esté en primera instancia; trato de apoyar a mis estudiantes, en lo posible ser comprensiva, sin llegar al punto de ser permisiva, tratando de escuchar siempre a la gente, sea en lo personal, sea en lo profesional, tratando de no prejuzgar, tratando de que la gente sienta que puede hablar, que puede ser ella misma, que puede expresarse con libertad y ser la persona que en el fondo realmente es".

Se empieza a vislumbrar en estos relatos elementos que configuran la educación del ingeniero. Al respecto Kohlberg, Power e Higgins (2008) afirman que la calidad de la enseñanza dependerá de que los educadores consideren la disciplina y otras cuestiones propias de las instituciones de educación como problemas humanos cuya solución requiere la participación de todos sus miembros y en este sentido se debe dar lugar a un enfoque más democrático y comunitario. Se hace necesaria una reforma pedagógica basada no en el avance tecnológico sino de los ideales morales de justicia, democracia y comunidad.

De este modo, Frisancho (2001) interpreta que en la educación se debe dar prioridad a los valores personales y sociales y a lograr que el alumno reflexione libremente en torno a estos valores, los asuma y posteriormente los lleve a la acción. Así mismo el autor señala que:

los valores juegan un papel central en las propuestas educativas, pues ayudan a fundamentar determinadas conductas que se reconocen como deseables por encima de otras $y$, en consecuencia, toda propuesta educativa se fundamenta en valores y trata de organizarse alrededor de ellos. (p. 14).

En este punto es importante señalar las reflexiones hechas por Bolívar (2005), quien manifiesta que la formación profesional universitaria tiene entre sus objetivos fundamentales formar profesionales competentes al servicio de la ciudadanía y, en consecuencia, debe responder a las expectativas y necesidades de la sociedad que aspira a contar con un desempeño adecuado de los egresados no solo en el ejercicio de las funciones inherentes a su profesión. Además el mismo autor argumenta que:

El objetivo de la formación ética que se debe impartir en las facultades universitarias es que los estudiantes sean personas con criterio, esto es, que 
tengan la capacidad de reconocer las variables a considerar en el momento de afrontar un problema de tipo ético y saber distinguir lo que está bien de lo que está mal y que estén dispuestas a optar siempre por la vía de una actuación correcta de acuerdo con su conciencia y apegados a los principios de verdad, libertad y justicia. (p. 118).

La ética tiene sentido en relación con los grupos humanos ya que es en ellos donde la persona estructura sus conceptos normativos y sus prácticas morales. La ética se construye mediante la intersubjetividad y alteridad de los individuos.

En el contexto kantiano la ética tiene como aspiración el bien del individuo en sí y de la comunidad en la cual el individuo interactúa con base en las acciones bajo la norma de las máximas universalizables y de la voluntad como ejercicio de la libertad. En este sentido, la ética es esencialmente social y su proceso de formación surge a partir de la universalización de las costumbres que mejoran las condiciones de bienestar de los grupos. No obstante, la sola declaración de los códigos de comportamiento no es suficiente para lograr una comunidad con cultura moral, es necesario que cada individuo haya interiorizado el imperativo categórico con el cual valorará sus acciones en función de su propio bienestar y el de su grupo. No puede entonces concebirse un individuo cuyas acciones estén determinadas a lograr su bienestar usufructuando el bienestar de la sociedad, ni tampoco esta puede determinar su bienestar a costa de alguno de sus miembros.

La práctica minera es un buen ejemplo de la relación entre lo social y lo individual. Es interesante analizar que la propiedad privada cede paso a la propiedad pública en lo referente a los bienes obtenidos del subsuelo. El Código Colombiano de Minas, Ley 685 de 2001, en el artículo cinco (5) expresa que

los minerales de cualquier clase y ubicación yacentes en el suelo o el subsuelo, son de la exclusiva propiedad del estado, sin consideración a que la propiedad, posesión o tenencia de los correspondientes terrenos, sean de otras entidades públicas, de particulares con comunidades o grupos. (p. 2).

Al analizar el fin de la ética como una construcción de la armonía y de la convivencia social como la gran aspiración de todos los grupos humanos, es posible encontrar un sustento a la legislación mencionada y fundamentar el principio ético según el cual el bien social prima sobre el bien particular.

\section{Factor de inspiración para hacer lo correcto siempre}

Esta frase idealista al parecer representa una representación utópica de la ética. Con relación a esto los estudiantes señalaron que "la ética es lo que impulsa al ser humano a siempre hacer las cosas correctas". Fundamentaron esta representación aduciendo que "la ética es tratar de hacer lo correcto siempre, tratando también de ver la parte moral" y teniendo en cuenta "los principios de lo bueno o lo que debemos hacer correcto pero en cuanto a nuestros pensamientos y nuestras convicciones". Así mismo argumentaron que la ética "es ese pensamiento que cada vez que estamos haciendo algo que conocemos que es indebido nos reclama ... en la conciencia, en la representación de saber actuar para las demás personas, saber que uno debe tener un compromiso con los demás y con uno mismo con el fin de que se hagan las cosas correctas".

Estas representaciones de los estudiantes coinciden con la de los docentes en el sentido de que estos consideran la ética como el soporte en el que deben apoyarse todas nuestras actividades para perseguir el bien de los demás haciendo lo correcto y causando el mínimo daño posible a las personas del entorno. Esta aseveración los docentes la argumentan expresando que "la ética debe ser la vía, el soporte en el cual se apoyen absolutamente todas nuestras actividades para tomar conciencia de orientarnos hacia el bien, hacia lo correcto, 
hacia tratar de ser felices, porque esa es la razón fundamental de ser del hombre, perseguir ser feliz, pero procurando causar el mínimo daño posible, tanto al otro como al mundo en que vivimos".

De este modo, se puede evidenciar que los participantes visibilizan la ética como un actuar, que en términos de Gonzáles (2005) se traduce en la siguiente afirmación: el ser humano se realiza, finalmente en y por la acción. La acción, entendida en su sentido más amplio y comprensivo como un componente holístico del hombre. Es decir, que la existencia del hombre se basa en la acción ya que él asegura su perfección en la perfección de su acción. Sin embargo, el mismo autor manifiesta que hay acciones que resultan destructivas, degradantes o despersonalizantes. Para que una acción sea personalizadora debe promover la realización del hombre en todas sus dimensiones. La acción plenamente humana debe transformar la naturaleza, perfeccionar al agente y facilitar la liberación.

\section{Conjunto de valores y principios para la}

\section{convivencia en sociedad}

Para un gran número de estudiantes la ética está relacionada con los valores que la persona tiene, los cuales son aprendidos del entorno. Relacionan la ética con la sociedad y la definen como una ciencia que les da a las personas ciertos parámetros que debe seguir para cumplir con las buenas acciones dentro de la sociedad.

Pareciera entenderse que la ética está directamente ligada con el amplio espectro de los valores humanos. Estos, según Ratas, Harmib y Simon (1967), son cualidades que dan sentido a la vida; en algunos casos estas cualidades son inclinaciones íntimas y personales; algunos autores las consideran instintivas, otros creen que son adquiridos y que se va perfeccionando con las diversas actividades de la vida. Se hace evidente en los relatos de los estudiantes cuando afirman que la ética se relaciona con los valores, señalando que: "la ética son valores que se relacionan con la humildad, la sencillez, la solidaridad, el respeto por los demás, la comprensión y la honestidad".
Es importante entonces resaltar que es deber de las instituciones de educación superior fomentar en los estudiantes el desarrollo de una visión y sentido ético para orientar su ejercicio profesional reflejando en sus acciones un conjunto de valores como los manifestados por los sujetos. De este modo y como lo advierte Schön (1992): "la preparación de los profesionales debería reconsiderar su diseño desde la perspectiva de una combinación de la enseñanza de la ciencia aplicada con la formación de una enseñanza a la reflexión en la acción" (p. 9). De esta manera se podría contribuir a la educación para la ciudadanía preparando profesionales competentes que posan el sentido ético de la profesión y que, en consecuencia, actúan con ética profesional, ya que el ejercicio profesional va a constituir la principal aportación ciudadana de estos profesionales (Cobo, 2003).

En este punto de la discusión cobra una importancia relevante la relación docente-estudiante como factor que puede llegar a favorecer o entorpecer la formación integral de profesionales y ciudadanos íntegros. Al respecto Vélez (2006) manifiesta que la relación del profesor y el estudiante debe ser una relación personal - didáctica, donde se adquieren y se refuerzan valores como la honestidad intelectual, la exactitud y el rigor, la curiosidad promotora del estudio, la constancia en el trabajo y la persistencia a pesar de las dificultades. Se resalta aquí la postura de Hortal (1995) al expresar que:

Enseñar ética no es lo único que se puede hacer desde el contexto universitario para favorecer la formación ética de los futuros profesionales. No hay nada que contribuya más a la formación de personas morales que el que estas crezcan, se formen y vivan entre personas morales. (p. 62).

Se puede concluir entonces que en las representaciones sociales de estudiantes y docentes se muestra la importancia de la ética como normas o reglas que deben ser tenidas en cuenta, tanto en 
la vida personal como en el desempeño profesional para regular las relaciones humanas en una sociedad o comunidad de tal forma que aseguren la convivencia y el orden entre los individuos, sin que los intereses particulares atenten contra los intereses comunes.

Desde la mirada de los docentes la ética se manifiesta en aplicar flexibilidad, comprensión, receptividad y buen juicio en el quehacer, de tal manera que la inflexibilidad de las normas debe ceder a la consideración de las personas, sin llegar a la permisividad, por lo cual, en el proceso educativo se debe dar prioridad a los valores personales y sociales y a lograr que el estudiante reflexione libremente en torno a estos valores, los asuma y posteriormente los lleve a la acción.

Para los actores educativos la ética está relacionada con los valores de los sujetos, los cuales son aprendidos del entorno. Relacionan la ética con la sociedad y la definen como una ciencia que les da a las personas ciertos parámetros que deben seguir para cumplir con las buenas acciones dentro de la sociedad.

En la medida en que el ser humano en su proceso formativo adquiere la conciencia de sus actos, podrá ejecutar acciones reguladas por la ética y la moral y desarrollará la capacidad de discernir y priorizar adecuadamente las situaciones críticas o de conflicto que se puedan presentar en las relaciones sociales con sus pares, de tal forma que se favorezcan las posibilidades de tomar decisiones en consenso tendientes a lograr soluciones justas y equitativas y no unilaterales.

Para terminar, es fundamental señalar que futuras investigaciones aborden la formación humanística y particularmente la ética en campos del conocimiento como la matemática, la física, la biología y otras áreas de la ingeniería, para que el profesional no se convierta en un simple productor $y$ reproductor de objetivos, sino que considere su formación vital como sujeto, el cual requiere no solo el aporte de aspectos científicos y técnicos sino de elementos artísticos, culturales, éticos y estéticos.

\section{FINANCIAMIENTO}

Articulo derivado de la investigación titulada "Formación humanística en educación superior: La ética en la Ingeniería de Minas", avalado por el Fondo de Investigación de la Universidad Francisco de Paula Santander (Colombia) y el Grupo de Investigación en Estudios Sociales y Pedagogía para la Paz -GIESPPAZ- (Categoría B COLCIENCIAS).

\section{REFERENCIAS}

Agüero, M. (2008). El reto de la formación profesional de los administradores. Contaduría y administración. México: Universidad Autónoma de México.

Bolívar, A. (2005). El lugar de la ética profesional en la formación universitaria. Revista mexicana de investigación educativa, 10 (24), 93-123.

Bonilla, E., \& Rodríguez, P. (2005). Más allá del dilemas de los métodos. Bogotá: Norma.

Cobo, J. (2003). Formación universitaria y educación para la ciudadanía. Revista de educación del Ministerio de Educación de España, 75(59), 359-375.

Congreso de la República (2001). Código Colombiano de Minas Ley 685. Recuperado de:

http://www.ani.gov.co/sites/default/files/ley_0685_ de_2001_codigo_de_minas.pdf

De Lira, B. (2006). Ciencia y humanismo en formación universitaria. México: Universidad Autónoma de Aguas Calientes.

Frisancho, S. (2001). Educación y desarrollo moral. Lima: Ministerio de Educación del Perú.

Gonzáles, L. (2005). Ética. Bogotá: El Búho.

Hortal, A. (1995). La ética del profesional en el contexto universitario. Bilbao: Universidad de Desto.

Kant, I. (2006). Teoría y práctica. Madrid: Tecnos.

Kohlberg, L.; Power, C., \& Higgins, A. (2008). La educación moral según Lawrence Kohlberg. Barcelona: Gedisa.

Muñoz, P., \& Gamboa, A. (2012). Escuela y educación política: una mirada a los imaginarios de actores educativos. Pedagogía y saberes, 1 (36), 57-68. 
Pérez, I. (2006). La formación humanista y la ética profesional. La Habana, Cuba: Universidad de Cienfuegos.

Ratas, L.; Harmib, M., \& Simon, S. (1967). El sentido los valores y la enseñanza. México: Hispanoamericana.

Restrepo, J.; Amésquita, M.; Arenas, J., \& Castañeda , I. (2006). Cómo formar en humanismo y no morir en el intento. Revista Lassallista de Investigación, 3 (1), 66-72.

Schön, D. (1992). La formación de profesionales reflexivos. Hacia un nuevo diseño de la enseñanza y el aprendizaje en los profesionales. Barcelona: Paidos.
Strauss, A., \& Corbin, J. (2002). Bases de la investigación cualitativa. Técnicas y procedimientos para desarrollar la teoría fundamentada. Medellín: Universidad de Antioquia.

Taylor, S. J., \& Bodgan, R. (1990). Introducción a los métodos cualitativos de investigación. Barcelona: Paidós.

Vélez, O. (2006). La relación profesor-alumno en el aula como espacio de formación ética en la universidad. Revista digital de investigación en docencia univesitaria, 2 (1), 2-21.

\section{(9) (1) (8) $\Theta$}

